\title{
Structural and dynamic characteristics of technological power unit for heat treatment of vermiculite concentrates
}

\author{
Anatolij Nizhegorodov ${ }^{1, *}$, Alexey Gavrilin², and Boris Moyzes ${ }^{3}$ \\ ${ }^{1}$ Irkutsk National Research Technical University, Department of Construction and Road-Making \\ Machines and Hydraulic Systems, 664074 Irkutsk, Russia \\ ${ }^{2}$ National Research Tomsk Polytechnic University, Division for Materials Science, 634050 Tomsk, \\ Russia \\ ${ }^{3}$ National Research Tomsk Polytechnic University, Division for Testing and Diagnostics, 634050 \\ Tomsk, Russia
}

\begin{abstract}
The considered electric furnace with a mobile base is the most innovative power unit providing energy consumption of $48 \ldots 52 \mathrm{~mJ} / \mathrm{m}^{3}$ during the process of vermiculite firing which has never been achieved before. New power unit was developed for all mechanical shortcomings of the test prototype were eliminated. By eliminating the resonating base plate and switching to the platform with forced excitation of its vibrations, the influence of fluctuations in the excitation frequency of the oscillatory system on its technological parameters, ensuring the duration of vermiculite heat treatment, has been eliminated. The activity of high sensitivity of the oscillations of the base platform to the change in friction, which appears during operation, is also completely removed. In addition, the problem of a controlled and regulated vibrational method of supplying raw materials to the furnace without the use of drum doses has been solved. It simplifies the design and cost of new power units, which are characterized by doubled productivity and dynamic balance.
\end{abstract}

\section{Introduction}

The research of technologies and equipment for expanded vermiculite production and products from it have been carried out from the 40s of the last century to the present, both in our country and abroad [1-6]. It indicates the importance of this product and the incompleteness of above-mentioned research.

In the early 2000s, in connection with the economic recovery that began in Russia, the activity of producers of vermiculite concentrates revived, the Kovdorsky mining and processing plant in the Murmansk region, which produced high-quality concentrates, began to operate. Raw materials became available. And in 2003, the first electric modular- trigger furnace started to operate.

\footnotetext{
* Corresponding author: nastromo_irkutsk@mail.ru
} 
The further development of new furnaces for that time during almost twelve years, including the use of so-called «zero» modules [7] and the use of advanced overhead heating systems [8], led to the achievement of the highest indicator of their efficiency - specific energy consumption of vermiculite firing depending on the used concentrate, equal to $170 \ldots 182 \mathrm{~mJ} / \mathrm{m}^{3}$. But this reached the limit, and this concept did not allow move over it.

The idea of developing a fundamentally different concept of power units for vermiculite firing with a mobile base plate, put forward by the authors, appeared in 2015 and turned out to be very productive. These units replaced the modular-trigger furnaces. A one-module prototype of such a furnace was designed and tested in 2016. It showed a specific energy consumption of $48 \ldots 52 \mathrm{~mJ} / \mathrm{m}^{3}$ during tests when firing of vermiculite of the Koksharovskoye field [9]. But when testing the shortcomings associated with the mechanical part of the furnace unit - its mobile base plate, were identified.

The purpose of the research is to create the alternative mechanisms and processes for maintaining platform vibrations to implement the vibrotransport effect and dosing of vermiculite concentrate, as well as to analyze the design and dynamic characteristics of these new systems.

\subsection{Furnace with the resonant base plate}

Figure 1 shows a single-module version of an electric furnace with a resonating base plate and a drum type vermiculite concentrate dispenser. It consists of an electric furnace an oscillating system formed by a base plate 1 with a total mass of all its elements $M$ and springs 2 and 3 with a total stiffness equal to $\mathrm{N} / \mathrm{m}$ :

$$
c_{\Sigma}=c_{1}+4 c
$$

where $c_{1}$ - spring stiffness 2 of the eccentric drive 4, $c$ - spring stiffness 3 .

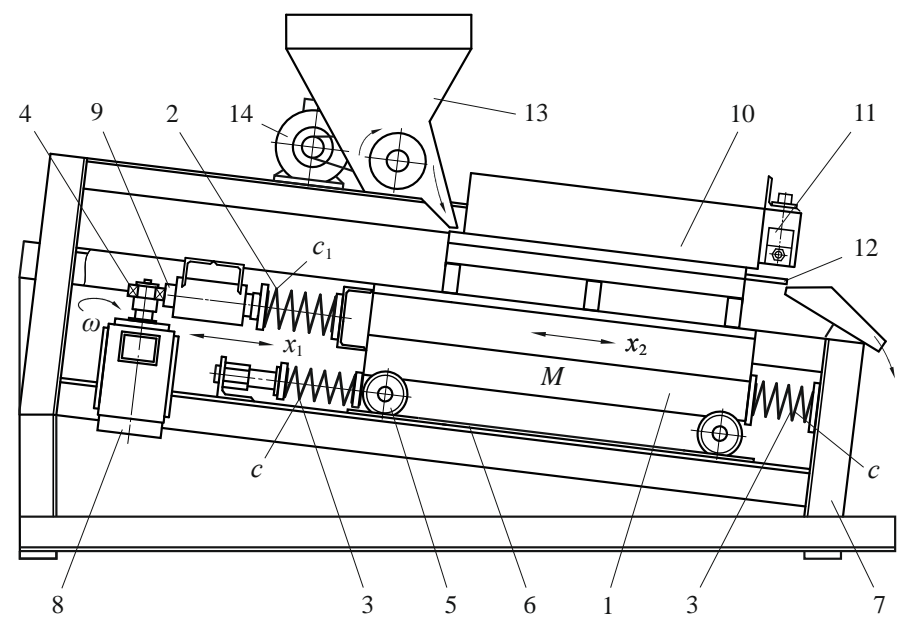

Fig. 1. A single-module version of an electric furnace with a resonating base plate

The platform is installed by its rollers 5 in the guides 6 of the frame of the furnace 7 and is driven into vibrational movement $x_{1}$ by an eccentric 4 with a frequency $\omega$ of the drive motor 8 through the pusher 9 and the mentioned spring 2 .

This dynamic system is linear. It has a high quality factor in the resonant mode of oscillations of the order of $20 \mathrm{~dB}$ [10] and this indicates its high quality. The resonance peak in such systems is almost symmetrical, narrow and elongated [11], but in our case this is a drawback. 
The oscillations of the base plate $x_{2}$, inclined at an angle of $19 \ldots 20^{\circ}$ to the horizon, provide a vibrotransport effect, i.e., the movement of the heat-treated vermiculite concentrate in the heat field of electric heaters which are located under cover 10 and held by mounting heads 11 of a single-module furnace unit (Fig. 1), during 2.8...3.2 s which are sufficient for concentrates with particle sizes from 3 to $6 \mathrm{~mm}$.

Figure 2 shows the amplitude-frequency characteristic (AFC) of the considered linear oscillatory system with the parameters of a single-module prototype:

$$
c_{1}=39876 \mathrm{~N} / \mathrm{m}, c=47970 \mathrm{~N} / \mathrm{m}, c_{\Sigma}=239756 \mathrm{~N} / \mathrm{m} ; M=105.4 \mathrm{~kg} .
$$

Moreover, its natural frequency, taking into account formula (1), is equal (rad/s):

$$
\omega_{0}=\sqrt{\frac{c_{\Sigma}}{M}}=47.7,
$$

and expressed equals $(\mathrm{Hz})$ :

$$
f_{0}=\frac{\omega_{0}}{2 \pi}=7.59
$$

The disadvantage of high-quality dynamic systems, both linear and non-linear is their high sensitivity [11-14]. Let's consider the ratio for the right side post-resonant branch of the frequency response $(\mathrm{dB} / \mathrm{Hz})$ :

$$
\frac{\Delta A}{\Delta f} \approx 3.7,
$$

where $\Delta A-$ is the amplitude increment at a change in the excitation frequency by $\Delta f$.

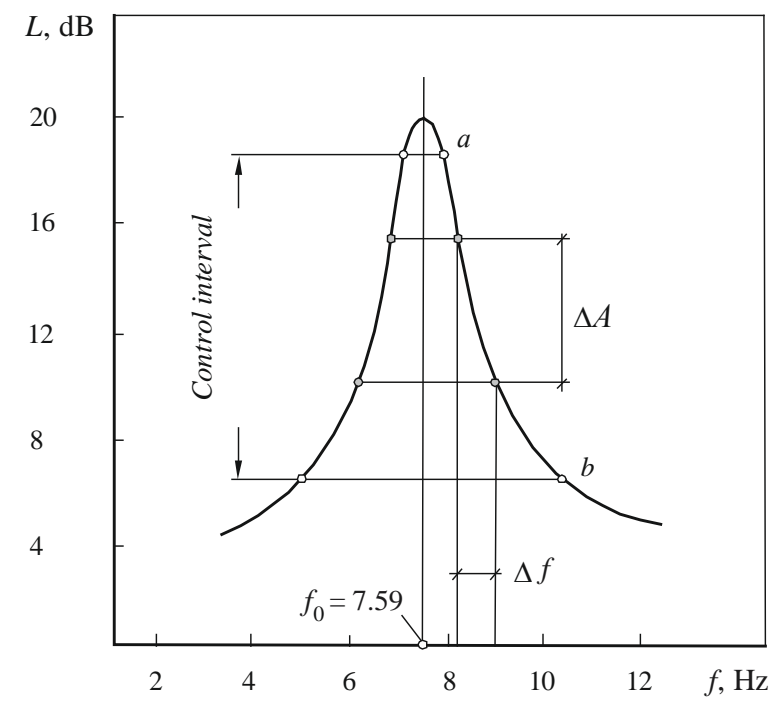

Fig. 2. Frequency response of the base plate

Since the resonance peak, as mentioned above, is almost symmetrical, for the left side below-preresonant branch this ratio is somewhat larger -4.3 .

Obviously, a slight fluctuation in the excitation frequency $f$ will lead to a significant change in the oscillations amplitude $A$ (Fig. 2) of vibration velocities $\omega A$ and vibration 
accelerations $\omega^{2} A$, which determines the intensity of the vibrotransport effect. In this case, it is the movement of vermiculite flow along the base plate 11 of platform 1 (Fig. 1). Therefore they determine the speed and time of firing of vermiculite concentrate. This is the technological parameters of the main process, deviation from which is unacceptable.

In nonlinear oscillatory systems, due to the tilt of frequency response, the sensitivity to fluctuations of excitation frequency is slightly less $(\Delta A / \Delta f=1.8 \ldots 2.5$ [15]), but this is still a lot, and to obtain strongly presented nonlinear oscillations using, for example, conical springs - impossible. Special and more complex elastic elements are required [16-18].

To reduce the sensitivity of the system, the operating point should be located as low as possible - in the region of point $b$ and below (Fig. 2). In this part, the amplitude of vibrations $A$ of the base plate of the furnace prototype during testing was about $2 \mathrm{~mm}$.

So, a furnace with a resonating base plate cannot provide stationary oscillations of the platform without any tracking system.

Another condition that make us to stop using the resonating platform is the significant sensitivity of the system to changes in friction, which was observed during testing of a single-module prototype shown in Fig. one

The rollers 5 are fixed on the bottom platform 1 and move together with the platform, rolling in the guides 6 . When firing vermiculite, a lot of finely dispersed material is released, which gradually settles on the frame guides. It leads to a gradual increase in friction and this reduces the high quality factor of the oscillating system and reduces the amplitude.

The damping coefficient of oscillations by friction is determined by the formula

$$
\xi=\frac{\alpha}{2 \sqrt{M\left(4 c+c_{1}\right)}}
$$

and for such dynamic systems on rolling bodies under conditions of good lubrication it is approximately $0.07 \ldots 0.075$ ( $\alpha$ is the coefficient forming the force of viscous resistance: $\left.\alpha \dot{x}_{2}\right)$.

Therefore, this is a high quality system but remains sensitive to changes in friction.

The deviation of the oscillation mode caused by friction leads to a decreased speed of vermiculite transportation on the base plate 12 and to an increased firing time. The result is the same - a change in the technological parameters of the firing process.

Another drawback which typically have modular-launch furnaces [7, 8] is the metering system with a drum dispenser 13 with a drive 14, Fig. 1, which feeds the raw material to the base plate 12 of the furnace. This system makes the furnace more complex and more expensive.

\subsection{Furnace with a forced base plate movement}

Above we discussed a single-phase version of the furnace, Fig. 1, which can be considered as a universal modular unit for industrial power units.

Fig. 3 shows the simplest coupled unit, consisting of two furnaces with a vertical layout of inclined modular units without drum dispensers.

It consists of a common frame 1, modular blocks, including a platform 2 with a base plate 3 , where the heat-treatable material run, a thermo-cover 4 , suspended electric heaters 5 , rollers 6 and tilted trays for removing expanded vermiculite 7. A hopper 8 for raw material concentrate is fixed on the frame with trigger trays 9, equipped with slide gate 10 .

The pressure of bulk materials on the bunkers walls defies the law of hydrostatic pressure, as liquids, and is evenly distributed along the height [17]; therefore, it is the same for both the upper and lower trays. 


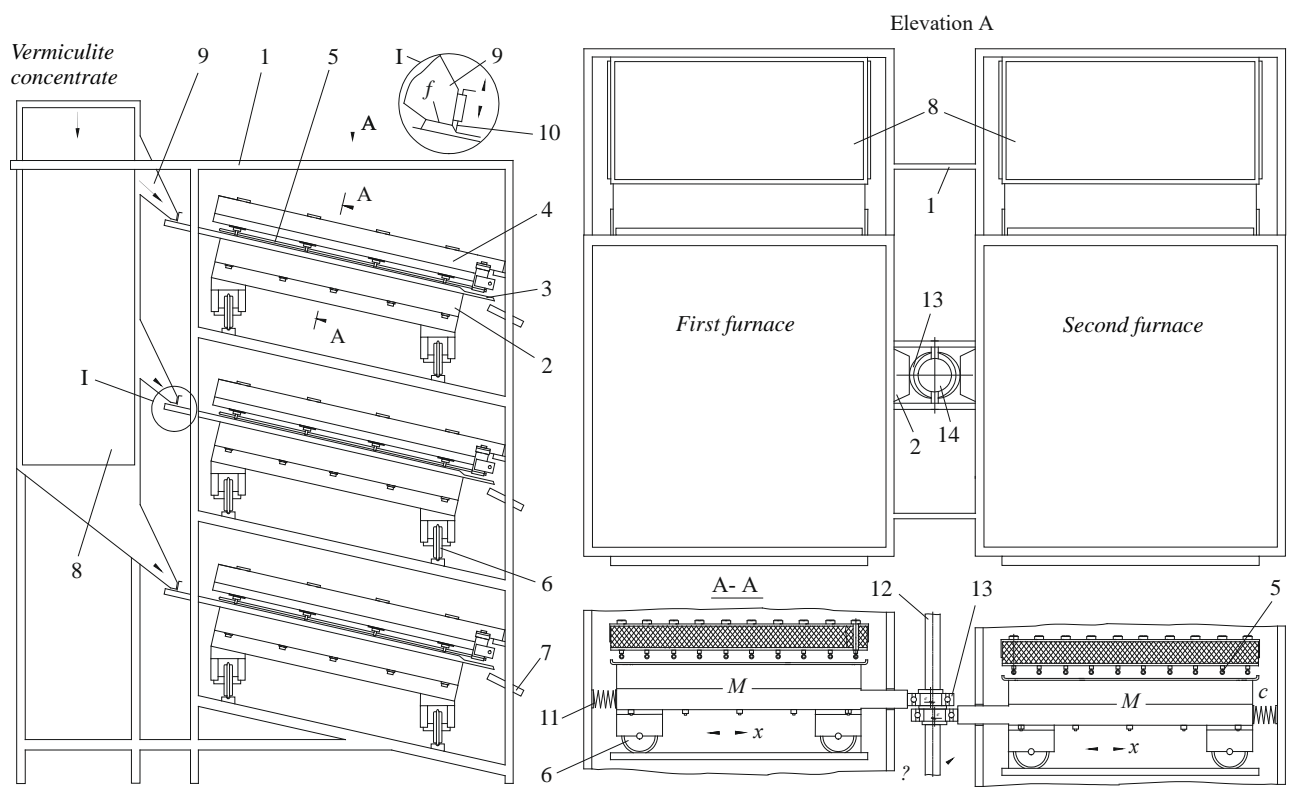

Fig. 3. Coupled power unit for the furnaces with vertical format

But, in order to eliminate its influence and reduce friction at the connection places with the base plates 3, the diversion trays 9 are elongated. A slight pressure from the particles of vermiculite concentrate on the base plate can be approximately determined by the formula:

$$
p=\frac{\rho \cdot V \cdot g}{f}
$$

where $\rho$ is the bulk density of the concentrate $\left(800 \ldots 1100 \mathrm{~kg} / \mathrm{m}^{3}\right), V$ is the material volume in the tray $\left(\mathrm{m}^{3}\right), f$ is the area of the outlet of the tray $\left(\mathrm{m}^{2}\right)$.

Assuming an average density value (950), volume $V$ (with a bottom plate width of $1 \mathrm{~m}$ ) equal to 0.018 and area $f$ equal to 0.04 , we obtain a pressure $p=4194 \mathrm{~N} / \mathrm{m}^{2}$. Then the friction force acting on the surface of the base plate during platform oscillations can be expressed as:

$$
F_{\mathrm{fr}}=p \cdot f \cdot \mu,
$$

where $\mu$ is the friction coefficient of vermiculite concentrate, approximately equal to $0.52[10]$.

Then, according to formula (6), the transversely directed friction force $F_{\text {fr }}$ on the base plate will be approximately equal to $87.2 \mathrm{~N}$. At this value, even when using a resonating base platform in the furnace, this force can be not considered. And in our case, it will not be able to influence the parameters of the oscillations of the base platform with forced movement.

The feeding of concentrate to the base plate 3 is controlled by gates 10 , which changes the thickness of the moving layer which coming out of the laying chute 9 . In this case, the feed must conducted in such way that the expanded grains of vermiculite move in a dense single-layer flow without gaps and layers. Otherwise, productivity will either be reduced or some of the material may not be fully expanded.

This method of feeding raw materials excludes the drum dispenser and its drive from the system and simplifies the design and cost. Taking into account the fact that the base 
platforms of the modules make oscillations transverse to the direction of vermiculite movement, it becomes possible to create a coupled power unit (Fig. 3).

The oscillatory system of the coupled power unit consists of a base platform with the total mass of all its structural elements $M$ and springs 11 , which provides only return movement, and the excitation of the base platform oscillations is set by a shaft 12 which is connected to the only drive motor in this design (not shown in Fig. 3) by means of bearings 13 which are eccentrically placed on the shaft.

The coupled design not only doubles productivity with one drive, but makes the entire unit dynamically balanced. Coupled eccentrics of the shaft 12 are made with a relative rotation of $180^{\circ}$, therefore, the inertia forces are balanced on each pair and don't affect the common drive shaft.

Since there is no elastic connection between the eccentrics and the base platforms, the movement of the left and the right platforms (see section A-A) is kinematically determined. Moreover, their oscillations are synchronous, antiphase and are described by a simple harmonic function:

- for left platform: $\quad x=-e \cdot \sin \omega t$;

- for right platform: $x=e \cdot \sin \omega t$.

Double time differentiation and multiplication by the mass of all elements of the base platforms gives the corresponding inertia forces:

$$
\begin{aligned}
& F_{1}=e \omega^{2} M \cdot \sin \omega t ; \\
& \quad F_{\mathrm{r}}=-e \omega^{2} M \cdot \sin \omega t .
\end{aligned}
$$

When the platform is in the extreme left position and its spring is fully compressed then inertia force reaches its maximum, it is directed to the left, and is equal to module

$$
F_{1 \max }=e \omega^{2} M .
$$

At this moment, the right platform is in the extreme right position and its inertia is directed to the right and is equal to

$$
F_{\text {r } \max }=e \omega^{2} M .
$$

Both forces are placed in common frame, which is a closed system, so inertial loads do not cause oscillations of the frame structure. When both platforms, oscillating synchronously and antiphase, "stand» in antiphase to themselves, then their maximum inertia forces in these positions are taken by eccentrics and the drive shaft and are closed on them. Here, vibrotransport can only be controlled by the frequency $\omega$ in the range from zero to the natural frequency, which is determined in this case by the formula, rad / s:

$$
\omega_{0}=\sqrt{\frac{2 c}{M}} .
$$

At $M=105.4 \mathrm{~kg}$ and a changed value of spring stiffness to $c=119800 \mathrm{~N} / \mathrm{m}$, we obtain the previous value of the natural frequency of oscillations -47.7 , so that the system would fully correspond to the tested prototype. If the excitation frequency is greater than the natural frequency $\omega>\omega_{0}$, then on each revolution of the shaft 12 (Fig. 3), there will be a separation, and then the impact of the platforms on the eccentrics 13 , so it is necessary to fulfill the condition: 
Returning to fig. 2, it can be noted that when testing a single-module prototype in the $\Delta \mathrm{A}$ range, the amplitude of the platform oscillations was approximately 2 to $3.2 \mathrm{~mm}$, therefore, the eccentricity $e$ should be taken equal to $3.5 \mathrm{~mm}$, and the setting for the required firing time (2.8...3.2 s) perform by changing the excitation frequency $\omega$.

Let's determine the power consumed by the vibrator to maintain the oscillations of one base platform at a natural frequency of $47.7 \mathrm{rad} / \mathrm{s}$, equal to the excitation frequency. At the viscous friction, the power dissipated during oscillations is determined by the expression (W) [11]:

$$
N_{\alpha}=0.5 \cdot \alpha e^{2} \omega^{2}
$$

Based on the formula (6), we determine the coefficient of viscous resistance $\alpha=692 \mathrm{~kg} / \mathrm{s}$. At $e=0.035 \mathrm{~m}$, the power $\mathrm{N}_{\alpha}$ is $964 \mathrm{~W}$.

We add the power loss to overcome the friction force (6) in the interface of the tray with the base plate. At one half-cycle, the negative work of $A_{\mathrm{fr}}$ of this force will be determined by the product of the force by displacement $(\mathrm{J})$ :

$$
A_{\text {fr }}=2 e F_{\text {fr }}=2 \cdot 0,035 \cdot 87,2=6.1 .
$$

When the oscillation frequency expressed in hertz is 7.59 (3), the platform will make twice as many full half-moves - 15.18, therefore, the additionally dissipated power will be equal to (W):

$$
N_{\text {ad }}=A_{\text {fr }} \cdot 15,18=92.6 \text {, }
$$

and the total $-1057 \mathrm{~W}$.

Given the loss in the bearings of the drive shaft through their efficiency (0.98), we obtain the value of the mechanical power consumed by one modular unit (W):

$$
N_{\text {mb }}=\frac{1057}{0.98}=1078.6
$$

For the entire coupled power unit, the power consumed by its mechanical systems will be six times greater $-6.5 \mathrm{~kW}$. The power of six electric heating systems is $135 \mathrm{~kW}$, so the part of the mechanical systems of the unit is only $4.8 \%$.

It is obvious that the energy consumption of the six drum dispensers eliminated from the design would be much greater, so the use of forced-running base platforms will also save energy.

\section{Conclusion}

The design of an electric furnace with a movable base platform is a forward-looking technological solution. After testing one of the samples of such a furnace, which showed a specific energy consumption of $48-52 \mathrm{~mJ} / \mathrm{m}^{3}$ during vermiculite firing, they replaced their precursors - electric modular-trigger furnaces. However, some disadvantages connected with dosing systems of vermiculite concentrates and expanded material movement in the firing space, forced to review some design features and significantly simplify them.

By eliminating the resonating base platform of the furnace and turning to the platform with forced kinematic excitation of its oscillations, the influence of fluctuations in the excitation frequency of the oscillatory system on its vibration velocity and vibration acceleration, which determine the intensity of the vibrotransport effect of the movement of the flow of expanded vermiculite in the thermal field of electric heaters, has been 
completely eliminated. In addition, the manifestation of a significant sensitivity of oscillations of the base platform of the furnace to the change in friction that occurs during its operation is eliminated.

The influence of these factors led to a change in the technological parameters of the material firing process - the speed of movement of the expanded particles and the firing time, which is unacceptable. So the first task was solved.

The second task, simpler and more obvious, it is the use of a controlled vibrational method of feeding raw materials to the furnace. It allows to exclude drum doses and their drives, significantly simplifies the design and cost of such furnaces, and taking into account the fact that the base platforms in the new electric furnace perform lateral vibrations, it becomes possible to create a coupled power unit which characterizes by doubled productivity, simple design and dynamic balance of coupled oscillatory systems - base platforms.

\section{References}

1. YU.M. Tikhonov, I.V. Kolomiets, A.S. Makbuzov, Stroitel'nye materialy: technology, 8 (2015).

2. YA.A. Akhtyamov, V.S. Bobrov, G.V. Gemmerling. Vermiculite firing. (Strojizdat, Moscow, 1973).

3. A.M. Rashad, Construction and Building Materials, 125 (2014).

4. D. Fan et al., J. of Industrial and Engineering Chemistry, 57 (2018).

5. S. Figueiredo, Chemosphere, 153 (2016).

6. L. Fuks, I. Herdzik-Koniecko, Appl. Clay Sci., 161 (2018).

7. A.V. Zvezdin, Vestnik ISTU, 8 (2015).

8. A.V. Zvezdin, A.I. Nizhegorodov, Vestnik ISTU, 1 (2019).

9. Rosnedra. On the Koksharovskoye field of vermiculite exploration operations have been completed URL: http://www.rosnedra.gov.ru/article/2132.html (19.09.2019).

10. A.I. Nizhegorodov, A.N. Gavrilin, B.B. Moyzes, Bulletin of the Tomsk Polytechnic University, Geo Assets Engineering, 3295 (2018).

11. Vibrations in technology: Handbook in $6 \mathrm{v}$. V. 1. Oscillations of linear systems. (Mashinostroenie, Moscow, 1979).

12. Vibrations in technology: Handbook in 6 v. V. 2. Oscillations of nonlinear mechanical systems (Mashinostroenie, Moscow, 1979).

13. W. Weaver, S.P. Tymoshenko, D.H. Young Vibration Problems in Engineering (Wiley-Interscience, New-York, 1990).

14. E. Halit. Acceleration, Vibration, and Shock Measurement (Taylor \& Francis Group, CRC Press LLC, Abingdon, 2000).

15. Dzh. P. Den Gartog. Mechanical vibration (Fizmat, Moscow, 1960).

16. A.I. Nizhegorodov, A.N. Gavrilin, B.B. Moyzes, A.I. Cherkasov, O.M. Zharkevich, G.S. Zhetessova, N.A. Savelyeva, IOP Conf. Ser-Mat. Sci., 2891 (2018).

17. A.A. Vajnson. Carrying and lifting machines (Mashinostroenie, Moscow, 1989).

18. A.I. Nizhegorodov, A.N. Gavrilin, B.B. Moyzes, T.B. Bryanskikh, A.V. Gradoboev, G.V. Vavilova, J. Tlusty, V. Tuzikova, Bulletin of the Tomsk Polytechnic University, Geo Assets Engineering, 3294 (2018). 